Portland State University

PDXScholar

$9-1-2011$

\title{
Whispering-Gallery Acoustic Sensing: \\ Characterization of Mesoscopic Films and Scanning Probe Microscopy Applications
}

Andres H. La Rosa

Portland State University, andres@pdx.edu

Nan Li

Portland State University

Rodolfo Fernandez

Portland State University

Xiaohua Wang

Portland State University

Richard Nordstrom

Portland State University

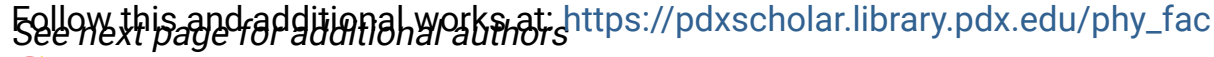

Part of the Physics Commons

Let us know how access to this document benefits you.

\section{Citation Details}

A. H. La Rosa, N. Li, R. Fernandez, X. Wang, R. Nordstrom, and S. K. Padigi, "Whispering-Gallery Acoustic Sensing: Characterization of Mesoscopic Films and Scanning Probe Microscopy Applications," Review of Scientific Instruments 82, 093704 (2011).

This Article is brought to you for free and open access. It has been accepted for inclusion in Physics Faculty Publications and Presentations by an authorized administrator of PDXScholar. Please contact us if we can make this document more accessible: pdxscholar@pdx.edu. 
Authors

Andres H. La Rosa, Nan Li, Rodolfo Fernandez, Xiaohua Wang, Richard Nordstrom, and S. K. Padigi 


\title{
Whispering-gallery acoustic sensing: Characterization of mesoscopic films and scanning probe microscopy applications
}

\author{
Andres H. La Rosa, ${ }^{1, a)}$ Nan Li, ${ }^{1}$ Rodolfo Fernandez, ${ }^{1}$ Xiaohua Wang, ${ }^{1}$ \\ Richard Nordstrom, ${ }^{2}$ and S. K. Padigi ${ }^{1}$ \\ ${ }^{1}$ Physics Department, Portland State University, P.O. Box 751, Portland, Oregon 97207, USA \\ ${ }^{2}$ Mechanical and Materials Engineering Department, Portland State University, P.O. Box 751, Portland, \\ Oregon 97207, USA
}

(Received 24 June 2011; accepted 18 August 2011; published online 19 September 2011)

Full understanding of the physics underlying the striking changes in viscoelasticity, relaxation time, and phase transitions that mesoscopic fluid-like films undergo at solid-liquid interfaces, or under confinement between two sliding solid boundaries, constitutes one of the major challenges in condensed matter physics. Their role in the imaging process of solid substrates by scanning probe microscopy (SPM) is also currently controversial. Aiming at improving the reliability and versatility of instrumentation dedicated to characterize mesoscopic films, a noninvasive whispering-gallery acoustic sensing (WGAS) technique is introduced; its application as feedback control in SPM is also demonstrated. To illustrate its working principle and potential merits, WGAS has been integrated into a SPM that uses a sharp tip attached to an electrically driven $32-\mathrm{kHz}$ piezoelectric tuning fork (TF), the latter also tighten to the operating microscope's frame. Such TF-based SPMs typically monitor the TF's state of motion by electrical means, hence subjected to the effects caused by the inherent capacitance of the device (i.e., electrical resonance differing from the probe's mechanical resonance). Instead, the novelty of WGAS resides in exploiting the already existent microscope's frame as an acoustic cavity (its few centimeter-sized perimeter closely matching the operating acoustic wavelength) where standingwaves (generated by the nanometer-sized oscillations of the TF's tines) are sensitively detected by an acoustic transducer (the latter judiciously placed around the microscope's frame perimeter for attaining maximum detection). This way, WGAS is able to remote monitoring, via acoustic means, the nanometer-sized amplitude motion of the TF's tines. (This remote-detection method resembles the ability to hear faint, but still clear, levels of sound at the galleries of a cathedral, despite the extraordinary distance location of the sound source.) In applications aiming at characterizing the dynamics of fluid-like mesoscopic films trapped under shear between the TF probe and the solid substrate, WGAS capitalizes on the well-known fact that the TF's motion is sensitively affected by the shear-forces (the substrate and its adsorbed mesocopic film playing a role) exert on its tip, which occurs when the latter is placed in close proximity to a solid substrate. Thus, WGAS uses a TF as an efficient transducer sandwiched between (i) the probe (that interact with the substrate and mesoscopic film), and (ii) the acoustic cavity (where an assessment of the probe mechanical motion is obtained). In short, WGAS has capability for monitoring probe-sample shear-force interactions via remote acoustic sensing means. In another application, WGAS can also be used as feedback control of the probe's vertical position in SPM. In effect, it is observed that when the microscope's probe stylus approaches a sample, a monotonic change of the WGAS acoustic signal occurs in the last $\sim 20 \mathrm{~nm}$ before the probe touches the solid sample's surface, which allows implementing an automated-control of the probesample distance for safely scanning the tip across the sample surface. This principle is demonstrated by imaging the topographic features of a standard sample. Finally, it is worth to highlight that this alignment-free acoustic-based method offers a very direct assessment of the probe's mechanical motion state (the mechanical and the WGAS acoustic frequency responses coincide), which makes the WGAS a convenient metrology tool for studying surface interactions, including interfacial friction at the nanometer scale. (C) 2011 American Institute of Physics. [doi:10.1063/1.3635224]

\section{INTRODUCTION}

\section{A. Whispering-gallery acoustic sensing (WGAS) in the context of other scanning probe microscopy (SPM) developments}

SPM groups a variety of proximal-probe imaging techniques able not only to characterize materials with nanome-

a)Electronic mail: andres@pdx.edu. ter scale resolution, ${ }^{1}$ but also getting involved in nanodevice fabrication. ${ }^{2}$ In a typical SPM, a sharp probe is scanned sideways along the sample's surface while keeping the probe's apex in close vertical proximity to the sample's surface, the latter requiring a precise control of the probe's vertical $z$-position to avoid crashing the tip against the sample's topographic features. SPM can be implemented to operate in either " $d c$ " or " $a c$ " mode. In the former modality, the probe is maintained in close proximity to the sample's surface (while the 
lateral scanning is taking place). That is the case in scanning tunneling microscopy ${ }^{3}$ and dip-pen nanolithography, ${ }^{4}$ for example, where the proximity of the probe to the sample is an essential requirement for the technique to work. However, such contact proximity requirement by the $d c$-mode approach presents some drawbacks when analyzing, for example, insulator materials for the unavoidable presence of charged impurities along the sample surface can convolute the SPM signal that controls the probe's vertical position. One way to overcome this shortcoming constitutes the $a c$-mode, where the probe is vertically dithered (i.e., intermittently placed closer to and away from the surface) thus minimizing the effect of local unwanted disturbances. In the $a c$-mode, the control of the probe's vertical position relies on the monotonic dependence of the probe's oscillation amplitude (as well as phase and resonance frequency shift) with the average probe-sample distance. The closer the tip gets to the sample's surface the smaller the probe's oscillation amplitude, which provides a guiding mechanism to implement a z-feedback control of the probe's vertical position in a $20 \mathrm{~nm}$ range near the solid substrate. This $a c$-mode is frequently used, for example, in atomic force microscopy. Controlling the probe-sample distance very reliably is fundamentally important in SPM.

However, some SPM techniques cannot afford dithering the probe vertically. That is the case, for example, in scanning near-field optical microscopy ${ }^{5}$ where such vertical motion in the proximity of the sample's surface could lead to intermittent crashes of the probe's optical aperture against the surface, in detriment to the probe's aperture morphology. This would produce an uncontrollable deformation of the probing optical near-field. Lateral dithering of the tip (i.e., parallel to the sample's surface) becomes then a preferred choice, with the probe's amplitude of oscillations monitored either via optical ${ }^{6,7}$ or electrical means, ${ }^{8}$ the latter is typically favored in order to avoid adding unrelated optical fields to the near-field optical measurement. Currently a piezoelectric tuning fork (TF), carrying a sharp probe stylus attached to one of its tines, is widely used due to its unique low damping properties, allowing its piezoelectric response to sensitively detect vibration amplitudes with sub-nanometer precision. ${ }^{9}$ In addition, because of the inherent mechanical rigidity of the experimental setup along the vertical direction, TF-based SPMs do not suffer from sudden snap-jumps of the tip towards the sample surface (due to capillary forces) as occur in AFM systems. The better reliability in controlling pre-determined tip-sample distances makes the TF-SPM a valuable metrology tool to study surface interactions. Such advantage has prompted to also implement TF-based SPM with vertical dithering. ${ }^{10,11}$

In TF-SPM the control of the probe's vertical position is implemented by electrically driving the TF with an ac voltage ( $\sim 1 \mathrm{mV} \mathrm{rms}$ ) and synchronously detecting its corresponding admittance. ${ }^{12}$ It turns out, however, that the reactance of the TF's inherently capacitance ends up convoluting the electrical detection of the TF's tines amplitude. This capacitance prevents the operator from determining the exact state of the TF's mechanical motion, including, for example, whether or not the probe has been brought to a complete rest by the interactions between the tip and the substrate; (even in that latter state the TF's admittance signal would be different from zero). Precise determination of whether or not the tip is at rest (as a consequence of its interaction with the solid substrate and the adsorbed mesoscopic fluid) provides pivotal information about the ability of shear forces to influence the probe's motion, an important aspect for friction studies at the nanometer scale. Even though alternative methods have been introduced to compensate these shortcomings, ${ }^{13}$ simpler methods to directly attest the real state of motion of the probe would be desirable.

WGAS, introduced in this manuscript, offers an alternative for establishing a direct correlation between the acoustic signal and the TF's mechanical motion, hence providing an opportunity to make a more direct assessment of tip-sample interactions when integrated into a TF-SPM. The acoustic detection approach circumvents the shortcomings associated to the TF's capacitance that arises in purely electrical detection settings. The simplicity of WGAS, as described in more detail in Secs. II-V, resides in using the existent microscope's frame as an acoustic cavity where standing waves (generated by the mechanical motion of the electrically driven TF) are detected in a whispering-gallery type modality; that is, similar to the faint, still clear, conversations that can be heard in the galleries of a cathedral from a source located at extraordinary distances. ${ }^{14}$ Exploiting constructive wave interference effects established in the acoustic cavity, WGAS can sense the TF's tines lateral motion down to nanometer-sized amplitudes. For a more comprehensive description of WGAS, this article is organized as follows: Section II details the experimental setup, which is basically a TF-based SPM slightly modified to integrate additional acoustic sensory components. Section III shows the experimental results that contrast the similarities, and differences, among the optical, electrical, and acoustic signals all of them used to monitor the mechanical motion of the TF. After demonstrating its sensitivity to nanometer sized changes in the TF's oscillations amplitude, WGAS is used to monitor the probe-sample interactions at various probe-sample distances. Section IV details the experimental arrangement to extend the use WGAS as position control feedback in SPM. Section V summarizes the advantages and necessary further investigation that can lead to an improved WGAS. Altogether, the results to be presented here should corroborate the advantage of acoustic techniques for studying fundamental surface phenomena at the nanometer scale.

\section{EXPERIMENTAL SETUP}

Figure 1 shows schematics of the experimental setup for acoustically monitoring the probe's mechanical motion in a TF-based SPM. (The adaptation of this WGAS setting as a feedback control for topographic imaging applications will be described later, in Sec. IV below.) Four main components can be identified: (i) a piezoelectric TF of $32 \mathrm{kHz}$ nominal resonance frequency and externally driven by a signal generator; (ii) an acoustic cavity (in mechanical contact with the TF); (iii) an acoustic transducer, located away from the TF but in mechanical contact with the cavity; and (iv) a lock-in based synchronous detection of the acoustic signal. 


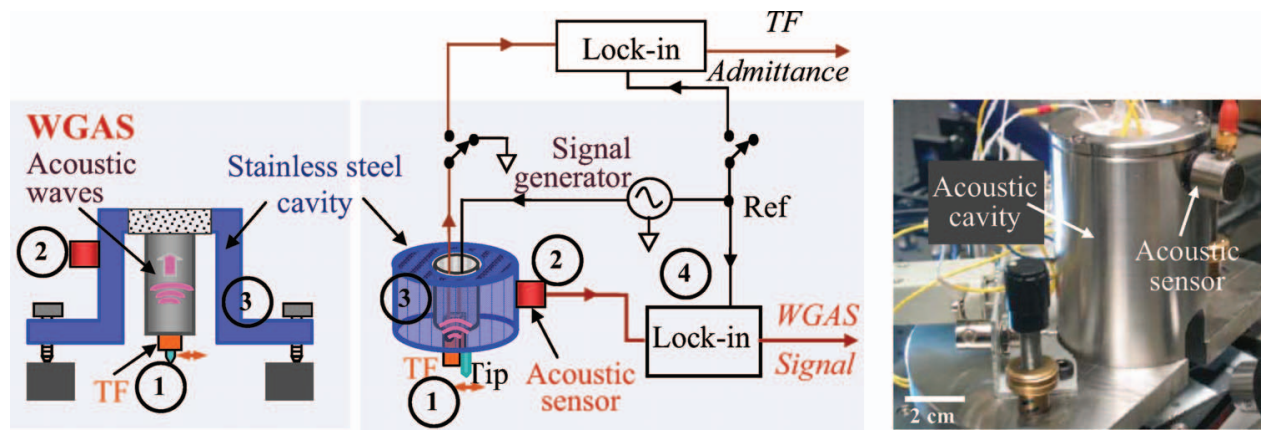

FIG. 1. (Color online) Left: Two schematic views of the WGAS experimental setting for sensing the mechanical vibrations of an electrically driven piezoelectric tuning fork (1). The mechanical waves engendered by the TF are detected by a remotely located acoustic sensor (2). The frame of a scanning probe microscope serves as an acoustic cavity (3). The acoustic waves are synchronously detected by referencing a lock-in amplifier to the TF driving frequency (4). The simultaneous detection of the TF's electrical admittance is optional. Right: Actual microscope frame of rounded shape, also serving as an acoustic cavity in the WGAS setup.

In a typical procedure, an ac-voltage $(\sim \mathrm{mV}$ rms amplitude) applied across the terminals of the TF makes its tines undergo lateral motion (against each other) of a few nanometers amplitude. The accumulated experimental results presented in this manuscript suggest (as it will be justified below) that the tight mechanical contact between the electrically driven TF and the acoustic cavity constitutes the source that engenders stationary acoustic waves into the cavity frame. Hence, placing an acoustic sensor at any of the acoustic nodes established around the periphery of the cavity maximizes the detected signal. The detection is implemented in a synchronous fashion using a lock-in amplifier. Optionally, and for control and comparison purposes, the TF's electrical admittance is also monitored simultaneously with the WGAS acoustic signal.

The piezoelectric $T F$ has two tines, each of dimensions $L=3.8 \mathrm{~mm}, t=0.6 \mathrm{~mm}$, and $w=0.35 \mathrm{~mm}$, with a spring constant $K=(E / 4) w(t / L)^{3}=26 \times 10^{3} \mathrm{~N} / \mathrm{m}$ (a Young's modulus $E=7.87 \times 10^{10} \mathrm{~N} / \mathrm{m}^{2}$ has been used in the calculation). Using a harmonic mechanical motion approximation and an equivalent RLC circuit fitting model, ${ }^{15}$ one obtains the piezoelectro-mechanical coupling $\alpha=6.5 \times 10^{-6} \mathrm{C} / \mathrm{m} .{ }^{16}$ At resonance (frequency $f$ and amplitude $u_{\mathrm{o}}$ ), the change in current is given by $\Delta I=4 \pi f \alpha u_{0}$, or $2.5 \mathrm{nA} / \mathrm{nm}$, a ratio that will be used herein to estimate the amplitude of the tine's amplitude of oscillation.

\section{A. The acoustic cavity}

Figure 1 also displays the rounded stainless steel frame in its double role (a) as the microscope's structural headstage, and (b) as an acoustic cavity. The dimensions of the cylindrical cavity are $158 \mathrm{~mm}$ external perimeter, $10 \mathrm{~mm}$ thick, and $75 \mathrm{~mm}$ tall; it is mounted on a truncated circular plate (150 $\mathrm{mm}$ diameter and $12 \mathrm{~mm}$ thick) with three finethreads screws (the latter for subsequent coarse positioning in microscopy operation applications). Notice in the figure the commercially available small-area acoustic transducer (DECI SE 25-P 426; 3 mm diameter sensitive area), positioned around the perimeter of the cavity. Its exact location is determined by the different nodes where the WGAS signal reaches a maximum (such locations vary, depending on the TF operating frequency). A very thin layer of vacuum grease helps to couple the cylinder's vibrations to the transducer. (Typically a plastic spring clamp, not shown in the figure, helps to keep a tight contact between the transducer and the cylindrical cavity.)

\section{B. Characterization of the cavity's mechanical response}

The picture at the top-left side of Fig. 2 shows the lower part of the acoustic cavity, highlighting the presence of a piezoelectric plate $(12 \mathrm{~mm} \times 12 \mathrm{~mm} \times 2 \mathrm{~mm})$ glued at the bottom of the supporting truncated circular plate. The purpose of the piezoelectric plate is to mechanically excite the cavity (at different frequencies and with the same amplitude) for recording its acoustic spectral response, particularly in a range around the $32 \mathrm{kHz}$ (the TF operating frequency). The corresponding WGAS experimental setting for this purpose is displayed on the right side of the figure. The spectral response, shown at the bottom-left side of the figure, is just one sample of more exhaustive measurements presented in Sec. III below. It can be inferred from this spectral analysis that, in general, an ideal cavity would be one that has a significant acoustic response at the particular TF operating frequency.
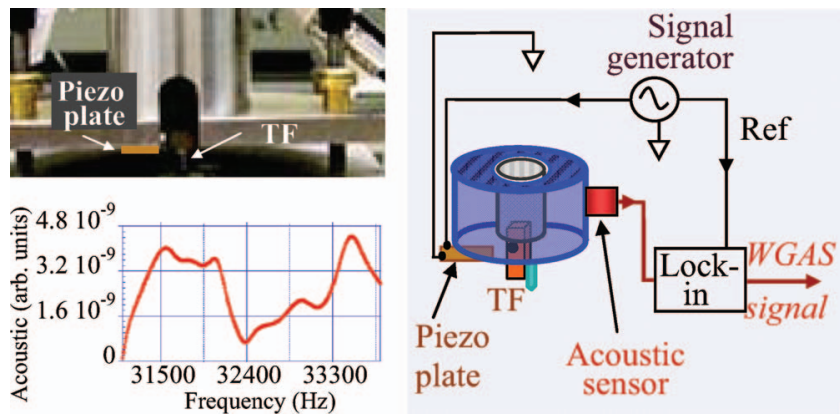

FIG. 2. (Color online) Frequency response of the acoustic cavity. The picture at the top shows a piezoelectric plate attached at the bottom of the cavity, which is used as excitation source (instead of the TF as in Fig.1). The diagram at the right shows the WGAS experimental setting used to acquire the cavity's acoustic response. The spectrum at the bottom-left was obtained keeping the acoustic sensor at an arbitrary fixed position and while driving the plate with $4 \mathrm{mV}$ rms. 


\section{Mechanical coupling between the TF and the acoustic cavity}

In the current WGAS setup (an extension of a scanning ultrasonic/shear-force near-field microscope (SUNM) hardware ${ }^{16}$ ) the acoustic cavity has a central axis that happens to be constituted by a piezoelectric tube $(20 \mathrm{~mm}$ diameter, $50 \mathrm{~mm}$ long) (see schematics shown in Fig. 1), but its role is just to hold the TF. Anticipating that a successful implementation of the WGAS will depend on a good mechanical coupling between the TF and the piezotube, a set of mechanical components were designed to (i) ensure a very tight mechanical contact between the TF and the piezotube, hence a tight attachment of the TF to the cavity, and (ii) preserve the integrity of the piezotube after repetitive loading/unloading the probe. These components are displayed in Fig. 3.

The optical image at the top shows a TF epoxy-glued to a holder (part-A) made out of Macor ceramic material. ${ }^{17}$ Macor is a material relatively easy to machine, and a good electrical and thermal insulator. Notice that the ceramic is designed to have two different outside diameters, one to easily fit into a conical-shape jacket (part-B) and the other a bit smaller to loosely fit the inner diameter of part-D. The image in the middle shows parts $\mathrm{B}, \mathrm{C}$, and $\mathrm{D}$, all made out of hard plastic material Delrin $\AA .{ }^{18}$ Part-B is a conical-shape "jacket" with partial incisions on one side as to form "leaves" that accept part-A with (initially) loose fitting. Part-C has one end permanently glued to the piezoelectric tube, while the other side is threaded on the outside diameter. Part-D is a cap of cylindrical shape with inner threads that fit part-C. When part-D is threaded on part-C it sandwiches part- $\mathrm{A}$ and part-B along the way, also causing the leaves of the "jacket" (part-B) to

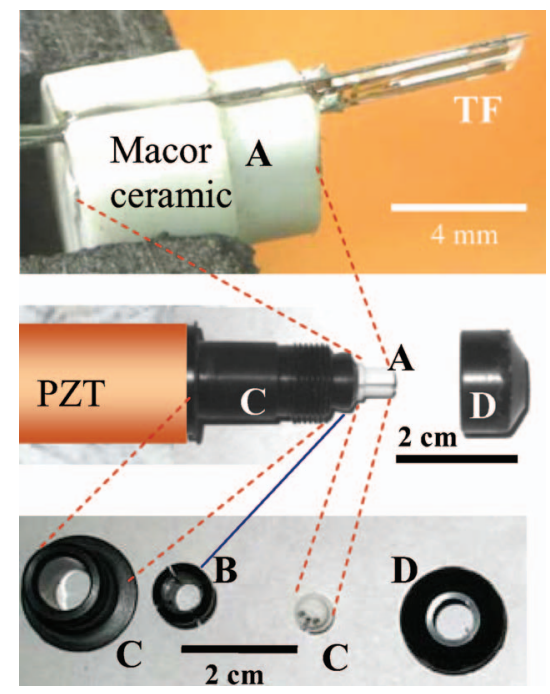

FIG. 3. (Color online) Components of the TF holder ensuring its tight mechanical coupling to the acoustic cavity. Top: Magnified view of a TF epoxyglued to a Macor-ceramic holder. Center: Side view of the components arrangement. Bottom: Top view of the different components. Part-A fits loosely into a conical-shape jacket B that has three "leaves" purposely cut. Part-C is permanently glued to a piezoelectric tube (PZT) on one end, and has a threaded termination on the other end for accepting part-D. When part-D is threaded into part-A the leaves of jacket B tight very well the Macor piece. This design ensures a repetitive loading and unloading of probes into the brittle PZT. tighten-sideways the Macor piece holding the TF. This design preserves the integrity of the piezoelectric tube, while ensuring a good mechanical contact with the tuning fork.

\section{Control and response variables}

Two variables are under experimental control: the tuning fork's driving voltage $\left(V_{d}\right)$, and the driving frequency $(f)$. Two signal responses are monitored, the WGAS acoustic signal and the TF's admittance (the latter for comparison purposes). The signal generator (a module inside a SRS-850) drives the TF supplying a programmable frequency $(f)$ and a programmable ac voltage amplitude $V_{d}$, (in the $4 \mathrm{mV}-10 \mathrm{mV}$ rms range). For detecting the acoustic waves set around the cavity, the acoustic transducer (DECI SE 25-P 426) is placed on the perimeter of the circular cavity (the microscope's frame) as indicated in Figures 1 and 2; different locations of the sensor around the microscope frame are tried first until a place of maximum signal is found. The response of the acoustic transducer is synchronously detected with a lock-in amplifier (SR850) set to current-mode detection and referenced to the TF driving frequency. For comparison purposes, the electrical admittance of the TF is simultaneously monitored with another lock-in amplifier (SR-844) also referenced to the TF driving frequency.

\section{EXPERIMENTAL RESULTS}

\section{A. Monitoring the frequency response of the TF}

Figure 4 shows the TF's mechanical response acoustically monitored by the WGAS modality while being electrically driven with $4 \mathrm{mV} \mathrm{rms} \mathrm{ac} \mathrm{voltage} \mathrm{and} \mathrm{using} 30 \mathrm{~ms}$ lock-in time constant (no tip was attached to the TF in this measurement). The acoustic experimental data (open circles) have been fitted (solid line) to the frequency-dependent amplitude response of a forced harmonic oscillator model using a damping constant $\gamma=2 \pi \times 6.5 \mathrm{~s}^{-1}$. (This gives a quality factor $\left.\mathrm{Q}=\omega_{0} / \gamma=\left(2 \pi \times 32062 \mathrm{~s}^{-1}\right) / \gamma \sim 5000\right)$. The inset in Fig. 4 compares the acoustic WGAS with the TF's electrical admittance signals near resonance; using such TF's

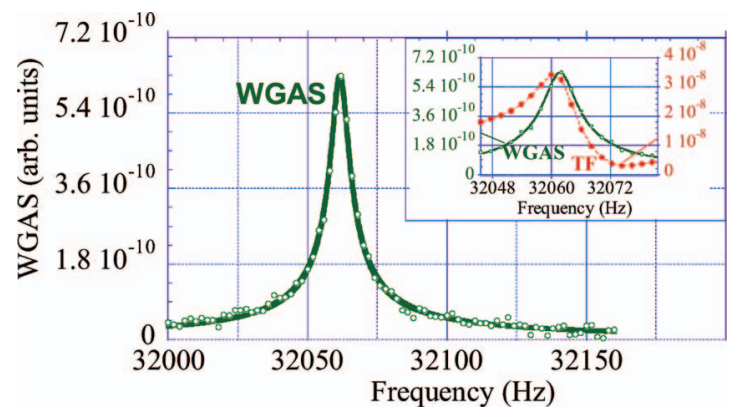

FIG. 4. (Color online) Magnitude of the TF's mechanical response acoustically monitored by WGAS. The solid line trace is a fitting curve to the experimental data (open circles) using the frequency-dependent amplitude response of a forced harmonic oscillator model. The inset compares the TF's mechanical response monitored by the acoustic WGAS and the TF's electrical admittance. The deviation of the electrical signal from the TF's mechanical response is caused by inherent capacitive character of the TF. 


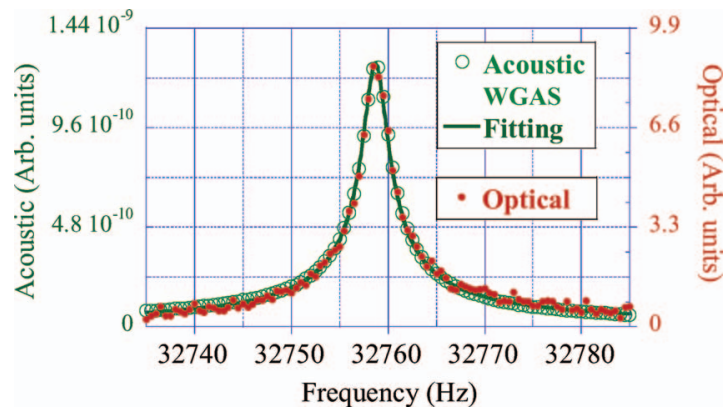

FIG. 5. (Color online) Mechanical response of a piezoelectric tuning fork monitored optically (filled circles) and acoustically by WGAS (open circles). The acoustic signal was fitted (solid line) to the amplitude response of a forced harmonic oscillator model.

admittance response and the conversion factor $2.5 \mathrm{nA} / \mathrm{nm}$, as indicated in Sec. II above, the amplitude of oscillation at resonance is estimated to be $9 \mathrm{~nm}$ in this particular case. Notice the difference between the TF admittance and the WGAS spectral response, putting in evidence the intrinsic capacitive character of the TF affecting the electrical measurement.

The acoustic WGAS reveals the TF mechanical response more directly. Supporting evidence of this claim is shown in Fig. 5, which compares the WGAS signal response with the optical monitoring of the tines motion, the latter acquired by focusing a laser beam on the edge of one of the TF's tines (using a lens of $5 \mathrm{~cm}$ focal length, and a $10 \mathrm{~mW} \mathrm{He-Ne} \mathrm{laser} \mathrm{op-}$ erating at $632.8 \mathrm{~nm}$ ) and detecting the optical diffraction pattern with a photodiode. This optical signal is synchronously measured with a lock-in referenced to the TF driving frequency. It is expected that the optical signal tracks directly the tines motion. The coincidence of the optical and WGAS acoustic spectral responses, acquired simultaneously, is evident in Fig. 5. The acoustic experimental data (open circles) have been fitted (solid line) to the frequency-dependent amplitude response of a forced harmonic oscillator model using a damping constant $\gamma=2 \pi \times 2.6 \mathrm{~s}^{-1}$.

\section{B. Frequency response of the acoustic cavity}

The WGAS detection setting was also used to examine the acoustic frequency response of the cavity, which is expected to be determined by its structural material composition (affecting the speed of the sound waves in the bulk) and geometry (due to the multiple internal reflections). The specific experimental setting was shown in Fig. 2 above. Of particular interest is the response in a range around $32 \mathrm{kHz}$ (the operating frequency of the typical TF used in this particular demonstration of the WGAS technique). For that purpose, an electrically driven piezoelectric plate (epoxy-glued to the base of the cavity, as shown in Fig. 2) was used to excite the cavity (4 $\mathrm{mV}$ driving voltage) at the different frequencies in the $28000 \mathrm{~Hz}$ to $34000 \mathrm{~Hz}$ range. (The voltage source driving the TF was turned off.) The resulting spectrum is shown by the trace with "open circles" in Fig. 6.

As a follow up test (trying to simplify the analysis of the somewhat complicated acoustic spectrum, as well as to identify the cavity's structural sections that may contribute more

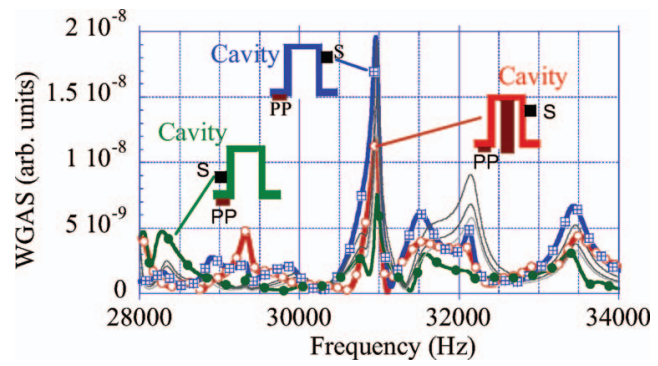

FIG. 6. (Color online) Frequency response of the microscope frame stage for different sensor settings. An electrically driven piezoelectric plate (PP) was used to mechanically excite the cavity, while the acoustic sensor (S) was kept at a fixed position during the acquisition of a given spectrum. Different spectra were acquired with the central axis of the cavity in place (trace with open circles) and without it (square and solid sphere traces). Multiple traces were obtained with the sensor at different arbitrary positions.

significantly to the frequency response) the central axis of the cavity (the piezoelectric tube alluded in Figs. 1 to 3) was removed, but the WGAS acoustic sensor was kept at the same position. The resulting acoustic spectral response is shown by the trace with "squares." Notice that for frequencies above 30 $\mathrm{kHz}$ there are no major differences between those two spectra (taken with and without the central axis), except that the peaks in the latter case are a bit higher. Subsequently, the sensor was placed at an arbitrary different position; the trace with "solid circles" shows the corresponding spectrum. Again, the major changes occur at frequencies below $30 \mathrm{kHz}$. The other thinner solid line traces correspond to other different tests performed at different times, and with the sensor located at arbitrary different positions. Overall, while some peaks appear in all the spectra at the same frequencies, there exist variations in the spectra depending on the position of the sensor.

The results presented in Fig. 6 suggest that, in order to operate WGAS, the resonance frequency of the TF (including the cases when a tip is attached to one of its tines) should be close to any of those values at which the cavity response peaks. This reasoning was supported by one further test (data not shown here) where, when using a TF of resonance frequency equal to $32650 \mathrm{~Hz}$, the detection of WGAS signal was very poor. However, after placing a small blob of mass on one of its tines (which moved the resonance frequency to $31450 \mathrm{~Hz}$ ) a WGAS signal was clearly detected. Hence, the different peaks shown in Fig. 6 represent the different operation frequencies available for implementing WGAS with this particular cavity.

\section{Monitoring probe-sample interactions (approaching curves)}

Upon the demonstration that WGAS is capable of monitoring the amplitude of the TF's oscillatory motion with nanometer sensitivity, it becomes clear that the new acoustic techniques can be used to monitor the shear force interactions between the TF's sharp tip and a solid substrate. Indeed, it is well known that when a sharp stylus is attached to one of the electrically driven TF's tines, the amplitude of oscillations is sensitively affected when the tip is brought in close proximity to a solid substrate. WGAS provides then an opportunity to monitor such shear-force interactions via acoustic means. 


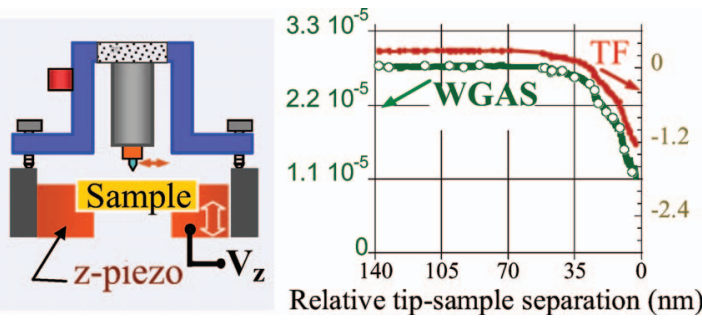

FIG. 7. (Color online) Variation of the WGAS signal (trace with open circles, in arbitrary units) as the sample approaches the tip (from left to right in the figure). The voltage $\mathrm{V}_{\mathrm{z}}$ applied to the z-piezoelectric element controls the relative tip-sample separation. For comparison, the variation of the TF admittance signal (arbitrary units) is also shown.

To test these ideas, a tapered probe is fabricated first out of glass fiber (3M FS-SC-6324) and by chemical etching procedures. ${ }^{19,20}$ The probe (typically a few millimeters in length, tapered region of $\sim 200 \mu \mathrm{m}$, and an apex termination of $\sim 100 \mathrm{~nm}$ radius) is attached to one of the tines of a TF. An atomically flat mica sample, serving as test substrate, is placed on a $z$-piezo stage (Piezosystem Jena, Tritor100 model; equipped with capacitance feedback capability to overcome piezoelectric hysteresis). Figure 7 displays the setting where $\mathrm{V}_{\mathrm{z}}$ voltage is applied to the piezo-stage to control the probe-sample distance. Notice also, as the tip gets closer to the sample (while the TF is being driven at its resonance frequency measured when the tip is far way from the substrate) no change in the WGAS signal is initially observed. But, when the tip is very close to the sample, a monotonic decrease in the acoustic signal is observed in the last $40 \mathrm{~nm}$. The reason for the decrease in the probe's amplitude of oscillation is usually attributed to the presence of a mesoscopic fluid-like layer (made out of water and other hydrocarbons molecules) that is typically found adsorbed to solid substrates at ambient conditions. Another hypothesis suggests that the cause is the intermittent probe-substrate contact. For comparison purposes, Fig. 7 also shows the TF's electrical admittance response.

Figure 8 displays the effects that the probe-sample interactions have on the probe's motion with some more detail. Variation of the acoustic signal is recorded as a function of time (trace with open circles) as the probe is placed successively closer to, or away from, a mica substrate (the tip remaining immersed or in contact with the adsorbed fluidlike layer most of the time). At the beginning $(t=0 \mathrm{~s})$ the tip is away from the surface. As the tip approaches the sample, neither the WGAS nor the TF signals change (see the flat shape of the traces around the instant "A"). Subsequently, both traces display a small decrease in signal intensity at the instant "B," which suggests that the tip has either just encountered the mesoscopic layer ${ }^{21}$ or, probably, the solid substrate. ${ }^{22}$ Afterwards the tip is forced to undergo brief trips towards to and away from the substrate. At the different instants "A," "C," and "D" the $\mathrm{V}_{\mathrm{Z}}$ voltage (used to control the relative probe-sample distance) was maintained at their corresponding fixed values, and a WGAS spectrum was recorded (see inset in Fig. 8). The spectra "C" and D" (tip positioned close to the substrate) reveal an increase in the probe's resonance frequency relative to the spectrum "A" (obtained when

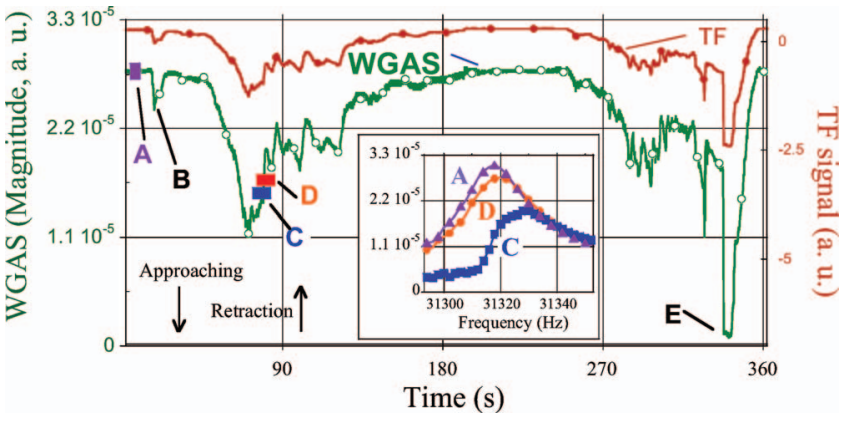

FIG. 8. (Color online) Time variation of the WGAS signal (trace with open circles) while the probe makes several short approaching and retraction steps $(<2 \mathrm{~nm})$ relative to a mica substrate. (At ambient conditions, a mesoscopic fluid-like layer typically covers the substrate, which likely affects the mechanical motion of the tip.) At " $\mathrm{A}$ " the probe is far away from the surface; subsequently the tip is immersed into the mesoscopic layer or in contact with the solid substrate. For comparison, the figure also shows the corresponding variation of the TF's electrical admittance (trace with filled circles), acquired simultaneously with the acoustic signal. The inset at the center shows WGAS spectra taken at three different instances, while interrupting momentarily the approach/retraction process. At "E," the WGAS (because of its direct correlation with the probe's mechanical motion) straightforwardly reveals that the TF is almost at complete rest (the zero scale of the WGAS is an absolute zero reference), which is not clearly discernable from the TF signal.

the tip was far away from the sample). The "blue-shift" revealed by spectrum "D" (contrary to an expected red-shift if the associated interaction were a purely damping force) may be attributed to the unusual properties of the mesoscopic fluidlike film mediating the interaction between the solid tip and solid substrate. ${ }^{16,21}$ Another potential source of the blue-shift is that the probe, while undergoing oscillatory motion (and because the probe, more likely, is not perfectly perpendicular to the substrate), is simply intermittently touching the solid substrate; this nonlinear interaction would cause not only a blue-shift of the resonance frequency but also a deformation of the bell-shape frequency response, ${ }^{23}$ which appears to be the case in spectrum "C" but not in the spectrum "D." A complete elucidation of the nature of these probe-sample shearforces is still lacking. The acoustic trace in Fig. 8 also reveals that at the instant "E" the probe is almost at complete rest, which is not directly discernible from the TF signal. In that regard, WGAS could provide a better metrology tool to investigate shear-force interactions since the WGAS scale constitutes an absolute scale directly related to the TF mechanical motion.

\section{WGAS AS A FEEDBACK POSITION CONTROL}

The monotonic behavior of the WGAS signal as a function of the tip-sample separation (as displayed in Fig. 7 above) provides a first straightforward indication about the feasibility for implementing acoustic-based feedback position control in SF-SPM. As the probe is laterally scanned across the sample surface, an eventual decrease of the probe-sample would produce a corresponding lower WGAS signal, an indication that the sample needs to be moved away to prevent an eventual crash of the tip against the sample. The change in the sample's z-position (to avoid the crash and maintain the same relative probe-sample distance) can be used to reconstruct the 


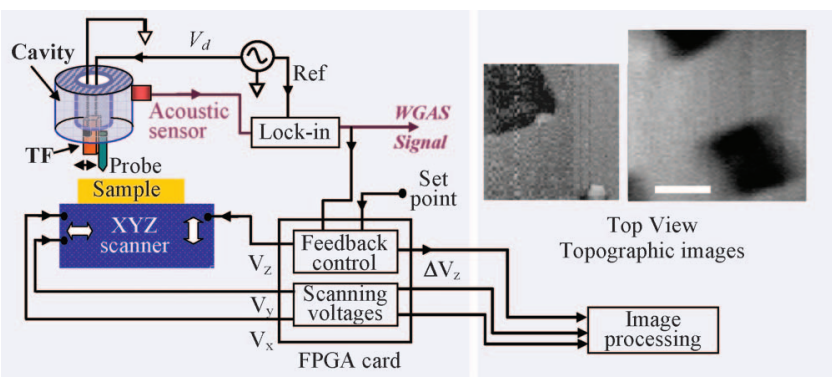

FIG. 9. (Color online) Top view of topographic images obtained using WGAS acoustic feedback probe-sample distance control. The scale bar in the right image is $5 \mu \mathrm{m}$.

sample's topography after multiple line scans across a specified sample region. The corresponding circuitry implementation of this feedback correction is shown in Fig. 9.

\section{A. The scanning stage}

Although a more compact implementation would result if the piezoelectric tube, shown in Fig. 1 as part of the central axis of the current acoustic cavity, was employed to scan the probe, we find that the scanning voltages applied to this piezoelectric tube detrimentally affects the WGAS signal. This observation supports the hypothesis that the acoustic signal, with its source at the TF, travels though the central axis of the stage towards the acoustic cavity; hence, scanning voltages applied to that piezoelectric tube would cause mechanical strains, thus convoluting the WGAS signal. As a consequence, the sample is scanned instead in all directions, while leaving the mechanical path from the TF to the acoustic transducer free of additional perturbations (see Fig. 9).

\section{B. Electronic station control}

We use a proportional integral differential (PID) feedback control designed in LABVIEW and implemented in a NI PCI-7831R real-time field-programmable gate array (FPGA) card module. ${ }^{24}$ It contains 8 independent analog inputs (4.0 $\mu$ s conversion time, 16-bit resolution, and $\pm 10 \mathrm{~V}$ sampling range) and 8 independent analog outputs (1.0 $\mu$ s update time, 16-bit resolution, and $\pm 10 \mathrm{~V}$ sampling range). The sampling and control voltages are digital and synchronized onboard (the latter has a resolution of $25 \mathrm{~ns}$ ). The LABVIEW control and data processing system work in a server and client mode. The NI PCI-7831R FPGA card is the server that provides (i) sampling of the TF and WGAS signals, (ii) control of the XYZ scanning voltages, and (iii) PID feedback control of the probe-sample distance; all of them independently. The LABVIEW software on the personal computer acts as a client, providing control and display interface to the user, and sends the service request from the user to the FPGA card.

\section{Imaging acquisition}

Upon the conversion of the acoustic vibration to an electrical signal (by the acoustic sensor transducer), the implementation of WGAS imaging application is quite similar to any other SPM. A set point voltage, selected by the operator, controls the probe-sample distance that the feedback system will try to keep constant while the sample is scanned laterally. As the tip encounters topographic ups and downs features during the lateral scanning, the feedback control produces the proper $\mathrm{V}_{\mathrm{z}}$ voltage corrections (and applied to the z-stage) to move the sample vertically and avoid crashing. A standard sample allows calibrating the $\mathrm{V}_{\mathrm{z}}$ voltage against the vertical z-displacement. For topographic imaging process, a fraction of the feedback high voltage $V_{z}$ as well as the $V_{x}$ and $\mathrm{V}_{\mathrm{y}}$ scanning voltages, are used to reproduce the sample topographic features. Figure 9 shows also a top view of the topographic images acquired using WGAS. The sample imaged is a micro-fabricated standard silicon sample that has a $200 \mathrm{~nm}$ thick thermally grown silicon oxide layer except at selected regions (squares of $5 \mu \mathrm{m}$ side). Notice that the regularly arranged topographic depressions are well imaged (dark regions in the figure). The images were acquired using $10 \mathrm{~ms}$ time constant in the lock-in amplifier setting, and at a scanning speed of 0.2 lines/s. The TF with the attached probe had a mechanical quality factor of $\mathrm{Q}=1000$.

\section{DISCUSSION}

The experimental results presented here provide further evidence of the sufficient sensitivity of acoustic detection methods for characterizing physical phenomena at the nanometer scale. Indeed, WGAS demonstrates that the interaction between a nanometer-sized solid probe and a solid substrate (see Fig. 7) can be monitored without perturbing the existent experimental setup of a TF-SPM as illustrated in Fig. 1, except of course using an extra acoustic transducer. But even so, the sensor ends up located at a distance far-away from the intervening interacting bodies; thus, in addition to tracking the probe's amplitude of oscillation with nanometer precision, such remote detection modality further highlights the noninvasive character of the new technique.

For these realizations, WGAS capitalizes on two main factors. First, the rigidity of the TF $(K \sim 20,000 \mathrm{~N} / \mathrm{m})$. The rigidity ensures that perturbations acting on the TF (the shear forces between the TF's sharp probe and the substrate, as illustrated in Fig. 7) are significantly coupled to the acoustic cavity; had the constituency of the TF be less sturdy, a perturbation of its motion would cause less impact on the acoustic cavity to which it is mechanically attached (hence, producing a much lower WGAS signal). Second, the resonance frequency of the TF (with a sharp probe attached to one of its tines) has to coincide with any of the many excitation frequencies (illustrated in Fig. 6) of the SPM frame (the latter acting as an acoustic cavity). Fortunately, the TF's resonance frequency, as well as the cavity frequency response, can properly be modified; the former by adding a small mass to the tines (as described in Sec. III above), the latter by a newly designed geometry (as proposed and detailed below).

Another advantage of using WGAS constitutes the straightforward information it reveals about the TF's state of mechanical motion, as evidenced by the results presented in Fig. 5. This can be particularly useful when monitoring shearforce interactions between a surface and a probe tip placed in 
close proximity (see Fig. 8) since, for example, a zero detection signal would imply that the probe has been brought to a complete stop. That inference can be made because the acoustic signal reflects directly the mechanical motion of the TF, thus providing an absolute scale of reference: no mechanical motion, no acoustic signal. This contrasts with the TF's electrical admittance detection method in which the condition of zero mechanical motion is not discernible straightforwardly (because the signal is convoluted with the contribution from the inherent TF's capacitance). This is also reflected by the different frequency response that the WGAS and the TF signals display, as shown in the inset of Fig. 4. Thus, despite the fact that the TF electrical admittance and the acoustic WGAS signals follow very closely each other (as shown in Fig. 8), the latter provides a more direct information of the TF's state of motion. This property may result quite beneficial in helping to understand friction phenomena at the nanoscale, where the conditions at which adhesion forces are able to bring the probe to a complete arrest needs to be determined with precision. On a broader context, WGAS could help contributing to a better understanding of the physics underlying the striking changes in viscoelasticity, relaxation time, and phase transitions that mesoscopic fluid-like systems undergo at solidliquid interfaces or when under confinement; these issues remain major challenges in condensed matter physics.

It is pertinent to also highlight that WGAS's implementation is very straightforward. It simply requires placing the transducer around the perimeter of an existent TF-based scanning probe microscope. Its working principle capitalizes on the presence of (previously overlooked) standing wave nodes of the microscope structure that, it turns out, can be sensitively detected by a commercially available acoustic transducer. The elaborated TF holder used in this first WGAS demonstration (see Fig. 3) responded to the desire of evaluating the new technique more systematically. But simpler holder designs may end up working equally well. On the other hand, although we proved the functioning of WGAS in an existent SPM (i.e., no modification of the frame stage was needed), an a priori design of a new microscope can take into account that a cavity with flat geometries on the outside perimeter would improve the coupling with the acoustic sensor. Cavities with hexagonal shape are currently being explored in our laboratory.

From an instrumentation development perspective, WGAS complements well the recently introduced SUNM, ${ }^{16}$ in which the dynamics of confined mesoscopic fluids under shear is monitored also via acoustic means. Thus, when studying the interaction between two sliding solid surfaces (a sharp probe tip and a substrate in this case) with a mesoscopic fluid trapped in between, the two techniques play a complementary role: (i) the SUNM monitors the effects that the shear interactions have on the dynamics of the mesoscopic fluid, while (ii) the WGAS monitors the effects on the probe.

It remains an open question to discern which specific part of the microscope frame geometry affects more effectively to the WGAS signal (just the cylindrical cavity, the flat plate supporter, or both?). These inquiries are important since they can lead us to find the ultimate sensitivity of the new technique, including, for example, whether or not it is able to measure the TF frequency response when excited by just the surrounding ambient thermal motion. Systematic test with modular frames (allowing analysis of its modular parts) could help to discern this question. This could be complemented with computer simulations, which can further guide a more optimum WGAS design towards developing a simpler, and noninvasive metrology tool for characterizing surface phenomena at mesoscopic scales.

\section{ACKNOWLEDGMENTS}

A.L.R. acknowledges support from the "2011 University Venture Development Funds" at Portland State University, Portland, Oregon; from the 2011 Milt Smith and Evelyn Kamback Fund foundation; and from the ONAMI TES Program funded by the U.S. Army through Grant No. W909MY10-C-0073.

${ }^{1}$ P. Eaton and P. West, Atomic Force Microscopy (Oxford University Press, New York, 2010).

${ }^{2}$ Tip-Based Nanofabrication, Fundamentals and Applications, edited by A. A. Tseng (Springer, Berlin, 2011).

${ }^{3}$ J. Chen, Introduction to Scanning Tunneling Microscopy (Oxford University Press, New York, 2007).

${ }^{4}$ K. Salaita, Y. Wang, and C. A. Mirkin, Nat. Nanotechnol. 2, 145 (2007).

${ }^{5} \mathrm{M}$. A. Paesler and P. J. Moyer, Near-Field Optics: Theory, Instrumentation and Applications (Wiley, New York, 1996).

${ }^{6}$ E. Betzig, P. L. Finn, and J. S. Weiner, Appl. Phys. Lett. 60, 2484 (1992).

${ }^{7}$ R. Toledo-Crow, P. C. Yang, Y. Chen, and M. Vaez-Iravani, Appl. Phys. Lett. 60, 2957 (1992).

${ }^{8}$ K. Karrai and R. D. Grober, Appl. Phys. Lett. 66, 1842 (1995).

${ }^{9}$ K. Karrai and R. D. Grober, Proc. SPIE 2535, 69 (1995).

${ }^{10}$ F. J. Giessibl, Appl. Phys. Lett. 73, 3956 (1998).

${ }^{11}$ R. C. Tung, T. Wutscher, D. Martinez-Martin, R. G. Reifenberger, F. Giessibl, and A. Raman, J. Appl. Phys. 107, 104508 (2010).

${ }^{12}$ K. Karrai and I. Tiemann, Phys. Rev. B 62, 13174 (2000).

${ }^{13}$ R. D. Grober, J. Acimovic, J. Schuck, D. Hessman, P. J. Kindlemann, J. Hespanha, and A. S. Morse, Rev. Sci. Instrum. 71, 2776 (2000).

${ }^{14}$ A. E. Bate, Proc. Phys. Soc. 50, 293 (1938).

${ }^{15}$ J. Rychen, T. Ihn, P. Studerus, A. Herrmann, K. Ensslin, H. J. Hug, P. J. A. van Schendel, and H. J. Guntherodt, Rev. Sci. Instrum, 71, 1695 (2000).

${ }^{16}$ A. H. La Rosa, R. Nordstrom, X. Cui, and J. McCollum, Rev. Sci. Instrum. 76, 093707 (2005).

${ }^{17}$ MACOR Machinable Glass Ceramic is a registered trademark of Corning Incorporated, Corning, NY. MACOR is composed of $\sim 55 \%$ fluorophlogopite mica and $45 \%$ borosilicate glass. Young's Modulus (at $25^{\circ} \mathrm{C}$ ) equal to $66.9 \mathrm{GPa}$, dc volume resistivity (at $25^{\circ} \mathrm{C}$ ) greater than $10^{16} \Omega \mathrm{cm}$.

${ }^{18}$ Delrin is the DuPont registered trademark. It offers a balance of desirable properties that bridges the gap between metals and ordinary plastics, with high mechanical strength and rigidity as well as good electrical insulating characteristics. Young's modulus $=2.5 \mathrm{GPa}$, dc volume resistivity $10^{13} \Omega \mathrm{m}$.

${ }^{19}$ P. Lambelet, A. Sayah, M. Pfeffer, C. Philipona, and F. Marquis-Weible, Appl. Opt. 37, 7289 (1998).

${ }^{20}$ R. Stockle, C. Fokas, V. Deckert, and R. Zenobi, Appl. Phys. Lett. 75, 160 (1999).

${ }^{21}$ C. L. Jahncke and H. D. Hallen, J. Appl. Phys. 93, 1274 (2003).

${ }^{22}$ K. Hsu and L. A. Gheber, Rev. Sci. Instrum. 70, 3609 (1999).

${ }^{23}$ M. J. Gregor, P. G. Blome, J. Schofer, and R. G. Ulbrich, Appl. Phys. Lett. 68, 307 (1996).

${ }^{24} \mathrm{X}$. Cui, "Near field acoustic microscope and its application in nano characterization," M.S. thesis, Portland State University, 2005. 